nervosa-namely, the low body weight, the resistance to eating, the admission that this had been a chronic condition, the lack of children, and the possibility of sexual difficulties in the marriage. Our patient would have been 25 years old when the war began, and it is possible that he already had chronic anorexia. His experience as a prisoner of war may have been used retrospectively to cover up the underlying psychiatric illness.

The aetiology of anorexia nervosa remains uncertain. Important life events may act as precipitants and have been found in the backgrounds of many patients with anorexia. The physical and psychological stresses among prisoners of the Japanese during the second world war have been described. ${ }^{1}$ The clinical signs in anorexia nervosa are due to a combination of energy and protein deficiency and could lead to severe cachexia. The predominant atrophy of type two muscle fibres seen in our patient was similar to that seen in women with anorexia nervosa and severe weight loss. ${ }^{2}$ Neuropathy and myopathy have also been described. ${ }^{3}$

Anorexia nervosa is often a relapsing and remitting condition lasting for many years. Our patient is of interest as there is little information about long term outcome in anorexia nervosa.

1 Tennant CC, Goulston K, Dent OF. The psychological effects being a prisoner of war: forty years after release. Am $\mathcal{f}$ Psychiatry 1986;143:618-21.

2 Lindboe CF, Askerold F, Slettebo M. Muscle changes in skeletal muscle of young women with Lindboe CF, Askerold F, Slettebo M. Muscle changes in ske

3 Alloway R, Reynolds EH, Spargo E, Russell GFM. Neuropathy and myopathy in two patients with anorexia and bulimia nervosa. I Neurol Neurosurg Psychiatry 1985;48:1015-20.

(Accepted 26 fanuary 1988)

Geriatric and Rehabilitation Unit, Blacktown Hospital, Blacktown, New South Wales 2148, Australia

N NAGARATNAM, MD, FRCP, geriatrician

D F GHOUGASSIAN, MB, BS, resident medical officer

Correspondence to: Dr Nagaratnam.

\section{Prosthetic valve endocarditis caused by Propionibacterium acnes}

We describe a case of recurrent endocarditis in a prosthetic mitral valve caused by an unusual organism-Propionibacterium acnes.

\section{Case report}

A 41 year old man was admitted to hospital with suspected infective endocarditis in April 1985. Three weeks earlier he had developed symptoms of a flu like illness, with malaise and sweating episodes; these followed infection of a finger prick wound, sustained when blood had been taken for measuring prothrombin time. His doctor had prescribed cephalexin $250 \mathrm{mg}$ four times a day for six days. Two days before admission he developed a swollen painful right ankle. In September 1983 he had undergone mitral valve replacement for rheumatic heart disease.

He was afebrile on admission. The prosthetic valve was functioning normally, and there were no murmurs. The only abnormal physical finding was a swollen erythematous right ankle. Three days later he developed an Osler's node on the left thumb, splinter haemorrhages, and an early diastolic murmur along the left sternal border. Endocarditis was diagnosed. Blood cultures were taken and treatment was started with intravenous ampicillin $2 \mathrm{~g}$ six hourly and gentamicin $80 \mathrm{mg}$ eight hourly.

The results of haematological and biochemical investigations were normal, as were those of serological tests for toxoplasmosis, brucellosis, cytomegalovirus, infectious mononucleosis, and autoantibodies. An initial echocardiogram was normal; a repeat echo cardiogram 10 days later confirmed aortic incompetence but showed no vegetations. Aerobic and anaerobic blood cultures were performed by the Bactec method. A positive signal was noted in the anaerobic cultures only after two weeks' incubation, and Propionibacterium acnes was identified in three consecutive cultures. It was sensitive to penicillin, ampicillin, cephalosporins, and gentamicin by disc diffusion tests. Minimum bactericidal concentrations of penicillin and ampicillin were 0.06 and $0.5 \mathrm{mg} / \mathrm{l}$ respectively.

Gentamicin was given for the first two weeks, and intravenous ampicillin was continued for four weeks. Serum bactericidal titres at one hour were $1 / 16$. He was discharged home well and told to take oral amoxycillin for a further two weeks. We have no doubt that he complied.

Five months later he returned to the outpatient clinic complaining of malaise and anorexia. The results of all investigations were normal and the echocardiogram was unchanged. Three sets of blood cultures were taken. All grew $P$ acnes from the anaerobic Bactec culture after two weeks. The results of antibiotic sensitivity tests were similar to those with the previous isolates. Readmitted for treatment, he was anxious, depressed, and had malaise and anorexia but no clinical signs of endocarditis. Penicillin $2 \mathrm{mU}$ four hourly, gentamicin $120 \mathrm{mg}$ eight hourly, and probenecid $500 \mathrm{mg}$ twice daily were prescribed. One hour serum bactericidal titre was $1 / 128$. Treatment was changed to oral penicillin $500 \mathrm{mg}$ four times daily plus probenecid after four weeks. The serum bactericidal titre was $1 / 64$. He was discharged home well. He continued taking oral penicillin for five months. He had no recurrence of endocarditis during 18 months' follow up.

\section{Comment}

$P$ acnes is a gram positive, non-spore forming, slow growing anaerobic $\mathbb{R}$ diphtheroid found on the skin. Its pathogenic role in acne vulgaris is established, but it has been rarely reported to cause systemic infection except in the presence of a prosthesis. ${ }^{1-3}$ Recently Fornaciari et al have described a case of $P$ acnes endocarditis. ${ }^{4}$ Our case is unusual because the probable source of infection was an infected pin prick site. The rarity of documented $P$ acnes infection may be due to the lack of good laboratory anaerobic culture techniques. Prolonged culture is also essential, and in this laboratory cultures from suspected cases of endocarditis are incubated for three weeks before being discarded. Despite the organism's sensitivity to the antibiotics a six week course of treatment failed to eradicate the infection. In the presence of a prosthetic device prolonged antibiotic therapy with high serum bactericidal concentrations may be required to prevent relapse of infection.

We thank Imelda Kenny for her secretarial help.

1 Whyte W, Hodgson R, Tinkler J, Graham J. Isolation of bacteria of low pathogenicity from faulty orthopaedic implants. I Hosp Infect 1981; Sept 2(3):219-30.

Calvin L, Strand MD, Richard E, Dubois MD. Propionibacterium acnes CNS shünt infections. Am $\mathcal{F}$ Clin Path 1981;75:743-6.

3 Levin J. Diphtheroid bactericidal endocarditis after insertion of a starr valve. Ann Int Med $1966 ; 64: 396-8$

4 Fornaciari G, Castagnetti E, Macari S, et al. A case of infectious Propionibacterium acnes endocarditis. Clin Ter 1985;115:389-92.

(Accepted 10 February 1988)

Department of Psychiatry, James Connolly Memorial Hospital, Blanchards town, Dublin

THERESE M O'NEILL, MRCPI, registrar

Mater Misericordiae Hospital, Dublin

ROSEMARY HONE, MD, consultant microbiologist

SEAN BLAKE, MD, FRCP, consultant cardiologist

Correspondence to: Dr Therese O’Neill, St Vincent's Hospital, Convent Avenue, Fairview, Dublin 3.

\section{Changing pattern of Crohn's disease in Northern Ireland}

Crohn's disease may well be a new disease of the twentieth century. ${ }^{1}$ Its incidence seems to be changing and has been described as increasing, ${ }^{2}$ decreasing, ${ }^{3}$ and having reached a plateau. ${ }^{4}$ We compared the incidences of the disease in two consecutive periods of eight years (1966-81) in a numerically stable population in a circumscribed geographical region.

\section{Patients, methods, and results}

Patients with Crohn's disease were identified from histopathology records and from hospital medical records; further epidemiological information was obtained from case notes. Patients were included in the study if they fulfilled strict criteria for a diagnosis of the disease $\mathrm{s}^{5}$ - that is, if they had two or more of: a clinical history of a combination of crampy abdominal pain, diarrhoea, and weight loss; typical macroscopic findings in the gastrointestinal tract at laparotomy or endoscopy; a definite diagnosis on histological examination of a resected or biopsy specimen; and characteristic radiological findings from contrast studies of the small or large bowel. Of 817 patients in whom Crohn's disease had been provisionally diagnosed clinically, 440 (183 men and 257 women) fitted these strict criteria. Thus the male:female ratio was $1: 1 \cdot 40$ compared with the ratio in the general population at the 1981 census of $1: 1 \cdot 04$

During 1966-73 the mean incidence of Crohn's disease in Northern Ireland was $1 \cdot 30 / 100000 /$ year $(1 \cdot 13$ for men and $1 \cdot 46$ for women), and during $1974-81$ it was $2 \cdot 34 / 100000 /$ year $(1.81$ for men and 2.85 for women). Thus from the first to the second period the incidence increased by a factor of 1.6 in men and 2.0 in women, or overall by a factor of $1 \cdot 8$. The number of new cases increased steadily from 1966 to 1981 . The distribution of cases by age at presentation showed a peak in the third decade in both men and women, a second peak in the sixth decade in women, and a preponderance of women in all but the seventh and ninth decades. 